Case Report

\title{
The Use of Etoposide, Ara-Cytarabine, and Melphalan (EAM) Conditioning Chemotherapy in Autologous Stem Cell Transplantation (ASCT) for a Patient with Relapsed Hodgkin's Lymphoma
}

\author{
Eko A. Pangarsa $\mathbb{D}^{1},{ }^{1}$ Ridho M. Naibaho $\mathbb{D}^{2,3}$ Vina Yunarvika $\mathbb{D}^{1},{ }^{1}$ Budi Setiawan $\mathbb{D}^{1}$ \\ Damai Santosa $\mathbb{D}^{1}{ }^{1}$ and Catharina Suharti $\mathbb{D}^{1}$ \\ ${ }^{1}$ Subdivion of Hematology and Medical Oncology, Medical Faculty of Diponegoro University, Dr. Kariadi General Hospital, \\ Semarang, Indonesia \\ ${ }^{2}$ Trainee in Hematology and Medical Oncology, Medical Faculty of Diponegoro University, Dr. Kariadi General Hospital, \\ Semarang, Indonesia \\ ${ }^{3}$ Department of Internal Medicine, Parikesit Hospital, Mulawarman School of Medicine, Samarinda, East Kalimantan, Indonesia
}

Correspondence should be addressed to Eko A. Pangarsa; ekopangarsa90@gmail.com

Received 1 August 2021; Accepted 15 October 2021; Published 3 November 2021

Academic Editor: Kostas Konstantopoulos

Copyright (c) 2021 Eko A. Pangarsa et al. This is an open access article distributed under the Creative Commons Attribution License, which permits unrestricted use, distribution, and reproduction in any medium, provided the original work is properly cited.

\begin{abstract}
Up to $20-40 \%$ of patients with Hodgkin's lymphoma will eventually relapse after treatment, among which early relapse confers a poor outcome. With salvage chemotherapy followed by autologous stem cell transplantation (ASCT), the longterm remission rate is $30 \%$. We report our experience of using a modified-BEAM conditioning regimen without BCNU consisting of etoposide, cytarabine, and melphalan (EAM) in a patient with relapsed Hodgkin's lymphoma. Before transplantation, the patient achieved second complete remission (CR2) using brentuximab vedotin and ESHAP (BRESHAP) chemotherapy. The ASCT went well without significant complications. This case demonstrated the considerable efficacy of EAM protocol as a conditioning regimen in terms of sufficient ablative capabilities, and the patient showed a successful hematopoietic engraftment. Although durability of the disease-free survival needs further observation, it had nearly 18 months of complete remission and the patient was in good performance status at the time of writing this manuscript.
\end{abstract}

\section{Background}

BEAM (carmustine (BCNU), etoposide, Ara-cytarabine, and melphalan) is considered as the standard conditioning regimen for autologous stem cell transplantation (ASCT) in malignant lymphoma [1, 2]. Unexpectedly, since 2010, the oncological community faced the issue of shortage of some essential chemotherapy drugs, among which was BCNU, one of the central components of the BEAM protocol. Physicians were, thus, forced to change their standard for those regimens in which a component was no longer available: two common solutions were to replace the missing drug with a substitutive agent or even to skip the unavailable drug, trusting that the modified regimen would yield a noninferior result in terms of efficacy and better toxicity profile [3].

BCNU shortage was also reported in Indonesia. So far, we have always been hindered to perform hematopoietic stem cell transplants by the unavailability of this particular drug in the national formularies [4]. We then employed a BEAM-like myeloablative protocol without the letter " $\mathrm{B}$ " consisting of etoposide, Ara-cytarabine, and melphalan (EAM) for a preparative ASCT regimen [5], of which one of the cases will be reported here in a patient with Hodgkin's lymphoma. 


\section{Case Presentation}

We report a young female patient with bone marrow "only" Hodgkin's lymphoma relapsed within 12 months after frontline ABVD chemotherapy; initial staging was unfavourable, Ann-Arbor II-B with International Prognostic Score (IPS) = 3. A previous pathological report (March 2017) from cervical lymphadenopathy revealed a mixed cellularity classical Hodgkin's lymphoma. Immunohistochemistry showed expression of $\mathrm{CD} 15+, \mathrm{CD} 30+, \mathrm{CD} 3+$, and high Ki67. The interim evaluation after treatment assessed by an 18-FDG PET scan is shown in Figure 1 and revealed a first complete remission (CR1) upon completion of 6 cycles of ABVD.

At relapse, she was staged as Ann-Arbor IV-B-E by PET/ CT scan (Deauville score 5) and showed bone marrow involvement "only." As salvage therapy, we treated her with ICE protocol showing partial remission (Interim PET/CT resulted in Deauville score 4) and then we switched to antiCD30 brentuximab vedotin in combination with ESHAP (BR-ESHAP regimen) for the second salvage. After 2 BRESHAP courses, examination by PET/CT demonstrated a negative metabolic activity of the bone marrow (Deauville score 2), see Figure 2.

She agreed to proceed to ASCT with her second complete remission (CR2) status. Peripheral blood stem cell (PBSC) harvest was performed in October 2019 by obtaining a CD34+ of $2.31 \times 10^{6}$ per $\mathrm{kg}$ of body weight. Our original uncontrolled rate freezing protocol with only $5 \%$ dimethyl sulfoxide (DMSO) by using the Planer 560-16 equipment (Planer products Ltd., UK) was used for stem cell cryopreservation, and the transplant was performed in December 2019. The cryopreserved stem cell was planned to be reinfused to the patient after EAM conditioning at least after 24 hours of free-chemo day. The EAM protocol consisted of intravenous etoposide (200 mg/m ${ }^{2}$ q24 hours) and Ara-cytarabine $\left(200 \mathrm{mg} / \mathrm{m}^{2}\right.$ q12 hours) administered for 4 consecutive days (days -6 to -3 ) followed by melphalan $140 \mathrm{mg} / \mathrm{m}^{2}$ given as a single dose at day -2 . The protocol and drug sequence administration are illustrated in Figure 3.

We summarized the transplant course day-by-day until hematopoietic engraftment, as shown in Figure 4. Following EAM chemotherapy and stem cell infusion, the ablative phase of transplant can be observed at day +2 . The absolute neutrophil count (ANC) began to decline to $<500 / \mu \mathrm{L}$ from day +4 , while platelet count kept going down to $<20,000 / \mu \mathrm{L}$ and reached its lowest point from day +5 . During transplantation, the patient is treated in a positive pressure isolation room and all supportive measures were adopted per local, including the administration of granulocyte colony-stimulating factor (GCSF) and infectious disease prophylaxis regimen. The posttransplantation period was uneventful though multiple packed red cells and platelet units were required to maintain hemoglobin concentration around $8-10 \mathrm{~g} / \mathrm{dL}$ and platelet count above $10,000 / \mu \mathrm{L}$ during this critical phase. No other significant nonhematological toxicities except nausea (grade 2), mucositis (grade 2), and diarrhea (grade 1) were reported. Neutrophil engraftment occurred at day +15 , and stable platelet count above $20,000 / \mu \mathrm{L}$ was noted from day +20 . With a follow-up of 18 months, the patient is in good health with complete and durable hematopoietic resolution with brentuximab vedotin was continued as the consolidation treatment after ASCT.

\section{Discussion}

Hodgkin's lymphoma accounts for approximately $10 \%$ of newly diagnosed malignant lymphoma [6]. Up to $20-40 \%$ of these patients will eventually relapse after treatment, among which early relapse confers a poor outcome [1, 7]. For patients who exhibited chemosensitivity, salvage treatment and then high-dose (conditioning) chemotherapy followed by ASCT is the current standard treatment $[1,2,6]$. We followed the methods of $\mathrm{Wu}$ et al. [8] utilizing brentuximab vedotin therapy to achieve clinical remission. Related to the conditioning regimen, the two randomized controlled phase III clinical studies with BEAM, mini-BEAM, Dexa-BEAM, or high-dose BEAM found that compared with conventional chemotherapy, salvage high-dose chemotherapy combined with ASCT can significantly increase the disease-free survival (DFS) of relapsed/refractory Hodgkin's lymphoma patients $[9,10]$.

There are several other commonly used regimens as conditioning chemotherapy for malignant lymphoma, namely, high-dose ICE (h-ICE; ifosfamide, carboplatin, and etoposide) [11], CMV (cyclophosphamide, melphalan, and etoposide) [12], CLV (cytarabine, lomustine and etoposide) [13], CBV (cyclophosphamide, BCNU, and etoposide), combination regimens including total body irradiation (TBI) $[7,12]$, rituximab or iodine-131-tositumomab combined with BEAM [14], and so forth. However, head-to-head comparisons of different conditioning regimens in relapsed/refractory lymphoma patients before ASCT are sparse.

Generally speaking, the BEAM regimen is currently recognized as the best conditioning regimen for Hodgkin's lymphoma $[15,16]$. The overall survival (OS) rate of the BEAM pretreatment regimen for Hodgkin's lymphoma patients is the highest with a 3-year progression-free survival (PFS) of $62 \%$ and a 3 -year OS of 79\% [17]. The BEAM regimen has also been compared to three-drug regimen such as CEB (carboplatin, etoposide, and bleomycin) or CLV (cytarabine, lomustine, and etoposide) regimen with better OS in favor of BEAM [13,18].

Many countries have also experienced temporary shortage of BCNU; the question is that could we simply omit the BCNU. A number of retrospective studies have tried to address the issue. Unfortunately, EAM conditioning in 12 Hodgkin's lymphoma and 11 non-Hodgkin's lymphoma patients by Loke et al. [19] gave disappointing results (mOS 29 months compared to 77 months for the matched BEAM group). A more recent study by Bekadja et al. [20] demonstrated comparable results in efficacy and toxicity with EAM, but the Ara-C total dose was $8 \mathrm{gr} / \mathrm{m}^{2}$. Other groups studied the replacement of BCNU in the BEAM regimen by 


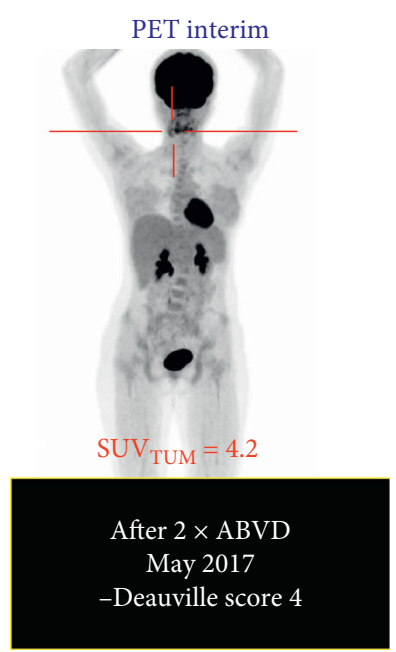

(a)

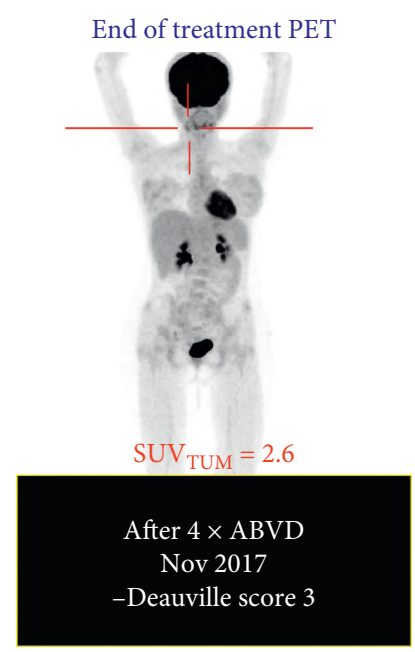

(b)

FIgUre 1: Clinical course of the patient after front-line treatment showing (a) PET-interim partial remission after induction ABVD chemotherapy; (b) PET/CT after completion of 6 cycles reveals complete remission and clinical improvement.

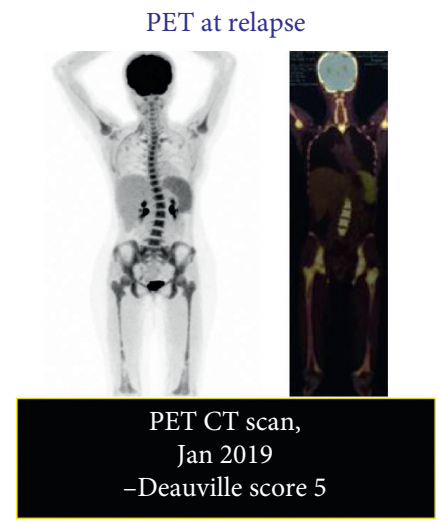

(a)

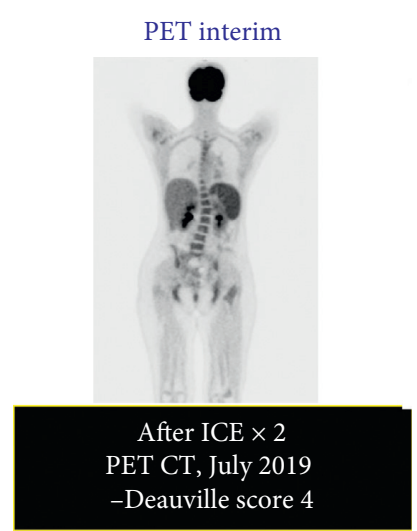

(b)

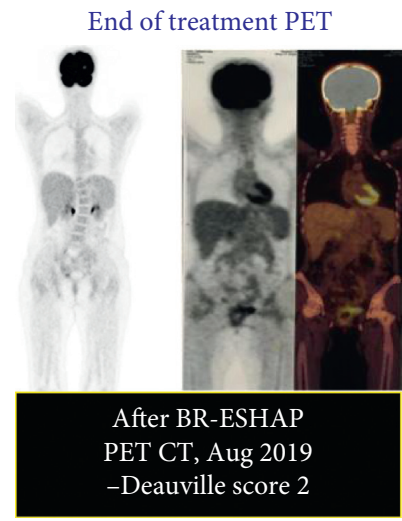

(c)

Figure 2: Clinical course of the patient at relapse. (a) First early relapse showing bone marrow progression (Ann-Arbor IVB-E); (b) partial remission after 2 cycles of the first ICE salvage treatment, with faint uptake over the bone marrow; and (c) complete remission after 2 cycles of second salvage using the brentuximab vedotin-ESHAP protocol.

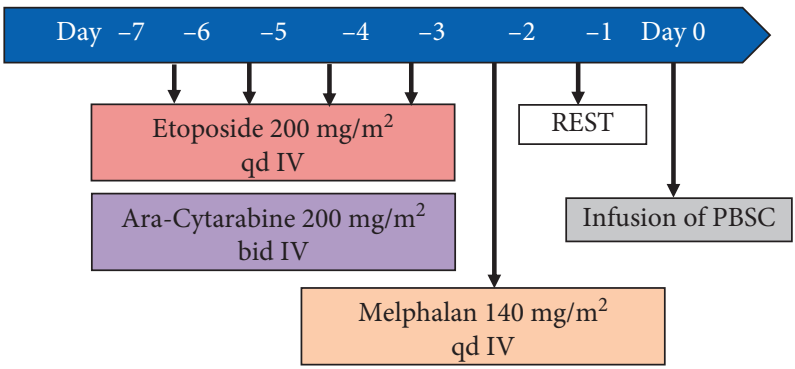

FIgUre 3: EAM protocol scheme at glance. Preharvested autologous stem cells were infused after 1 full day rest without chemotherapy (day zero). BMT, bone marrow transplantation; PBSC, peripheral blood stem cell.

lomustine or thiotepa and stated that although the BEAM group showed a lower rate of transplant-related mortality at day 100 , the difference did not reach statistical significance and the 3 regimens had similar results in terms of toxicity and efficacy [21].
This case describes our attempt at modifying the conditioning regimen for relapsed Hodgkin's lymphoma. In the absence of the BCNU component of the BEAM (BCNU, etoposide, cytarabine, and melphalan) $[15,22]$ or BEAC (BCNU, etoposide, Ara-cytarabine, and cyclophosphamide) 


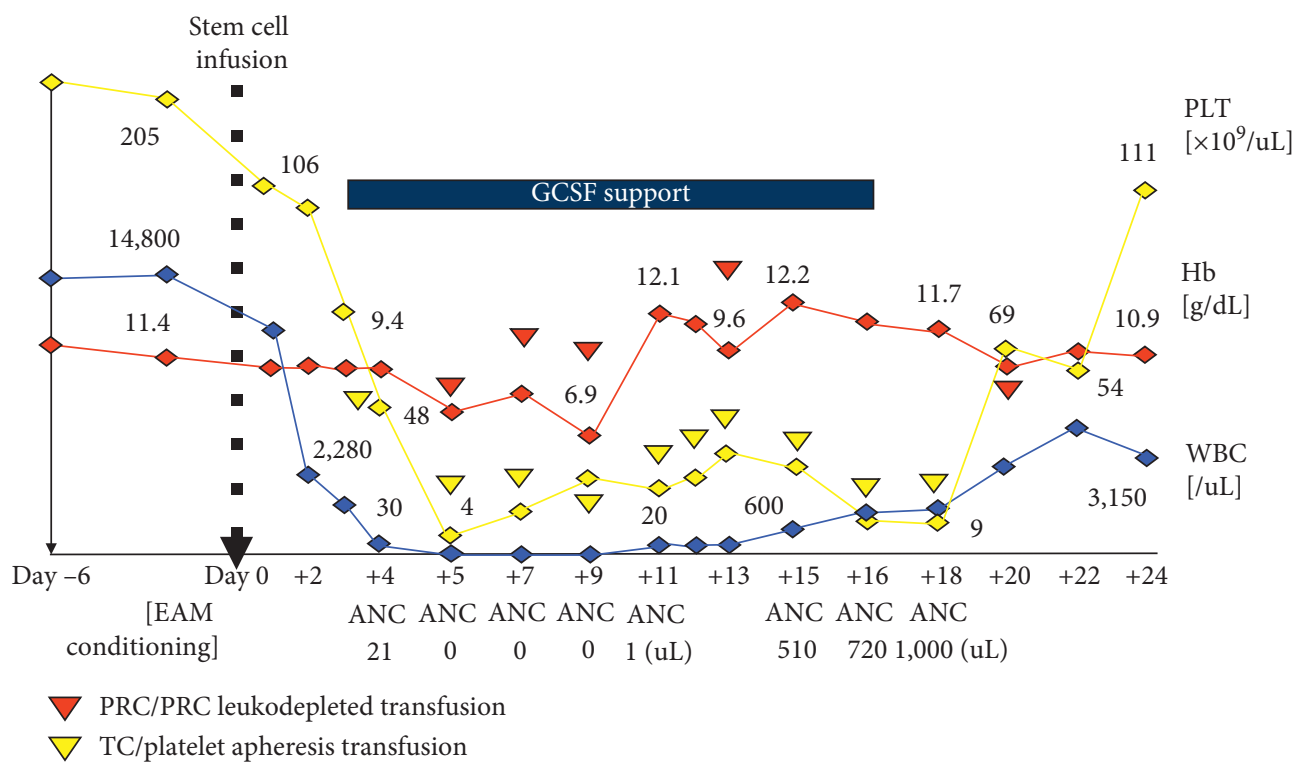

FIgURE 4: Day-to-day transplant evaluation from conditioning chemotherapy until engraftment. ANC, absolute neutrophil count; Hb, hemoglobin concentration; Plt, platelet count; PRC, packed red cells; and WBC, white blood cell count.

[14], the second most important consideration was drug availability while still maintaining effectiveness. The regimen should facilitate a successful transplant, create enough space for preharvested stem cell to regrowth, repopulate, and restore hematopoietic reconstitution or engraftment.

There are several adverse prognostic factors identified on patients with relapsed Hodgkin's lymphoma, among which others are short duration of CR after initial treatment [6]. The five-year-survival rates for early-relapse patients in the German Hodgkin Study Group (GHSG) cohort were below $50 \%$ [23]. In this patient, short remission duration $(<12$ months) was also reported along with presence of $\mathrm{B}$ symptoms, extranodal disease, and stage III/IV as predictors for another relapse post-ASCT [24]. A possible explanation for the favorable result of ASCT in the AETHERA study might be due to exclusion of poor-risked patients with refractory disease, good performance status, young age, sufficient stem cell harvest, and no life-threatening toxicity on salvage treatment. According to these clinical pre-ASCT risk factors, we planned to continue brentuximab vedotin up to a total of 16 cycles for better 5-year PFS based on the AETHERA study [25].

In this case report, we ablated the BCNU from the original BEAM regimen without altering either the dosage or drug sequence of the rest component, identical to Loke et al.'s protocol [19]. The details of our protocol can be found elsewhere [5]. Engraftment can be observed in day +15 and day +20 for neutrophils and platelets, respectively. Overall, the EAM scheme was well tolerated. As expected, nausea, mucositis, and diarrhea were the most prominent nonhematologic toxicity and fortunately manageable. These complications occur in frequent occasion with high-intensity regimens [13-23]. Considering the effectiveness of this three-drug regimen in longer-term outcome for PFS or even OS cannot be simply answered from this single case description. But, as depicted in Figure 4, the patient tolerated the expected ablative phase following the EAM regimen, and the cryopreserved stem cells successfully reconstituted hematopoietic engraftment without significant complications. We should admit that the utilization of brentuximab vedotin as the maintenance agent has made a significant survival advantage after ASCT as she is still in good condition until today.

\section{Conclusions}

We report a case of early-relapsed Hodgkin's lymphoma that underwent ASCT following CR2. The main message of this report is that the EAM protocol has the potential to be successful and effectively used in countries with limited access to BCNU. EAM was sufficient in terms of ablative capability to produce space for autograft to restore the hematopoiesis following ASCT. In the meantime, ASCT has not been widely carried out in Indonesia for several reasons; thus, this publication is needed at least to hand in a simpler and feasible conditioning protocol. Further noninferiority trial and prospective study could be specifically designed to provide both a more powerful evidence and survival outcome on the use of EAM in broader clinical practice.

\section{Abbreviations}

ABVD: Adriamycin, bleomycin, vinblastin, and dacarbazine

ANC: $\quad$ Absolute neutrophil count

ASCT: Autologous stem cell transplantation

BCNU: Bis-chloroethyl nitrosurea

BEAC: $\quad$ BCNU (carmustine, etoposide, cytarabine, and cyclophosphamide)

BEAM: $\quad$ BCNU (carmustine, etoposide, cytarabine, and melphalan) 


\begin{tabular}{|c|c|}
\hline CEB: & $\begin{array}{l}\text { Cyclophosphamide, etoposide, and BCNU } \\
\text { (carmustine) }\end{array}$ \\
\hline CBV: & Cyclophosphamide, BCNU (carmustine), and \\
\hline CLV: & Cyclophosphamide, lomustine, and etoposide \\
\hline CMV: & Cyclophosphamide, melphalan, and etoposide \\
\hline CR: & Complete remission \\
\hline DFS: & Disease-free survival \\
\hline DMSO: & Dimethyl sulfoxide \\
\hline EAM: & Etoposide, Ara-cytarabine, and melphalan \\
\hline G-CSF: & Granulocyte colony-stimulating factor \\
\hline GHSG: & German Hodgkin study group \\
\hline ICE: & Ifosfamide, carboplatin, and etoposide \\
\hline IPS: & International Prognostic Score \\
\hline ESHAP: & $\begin{array}{l}\text { Etoposide, methylprednisolone, cytarabine, and } \\
\text { cisplatin }\end{array}$ \\
\hline PBSC: & Peripheral blood stem cell \\
\hline 18-FDG & Positron emission tomography 18 \\
\hline PET: & $\begin{array}{l}\text { fluorodeoxyglucose positron emission } \\
\text { tomography scan }\end{array}$ \\
\hline PFS: & Progression-free survival \\
\hline TBI: & Total body irradiation \\
\hline OS: & Overall survival. \\
\hline
\end{tabular}

\section{Data Availability}

The data supporting the findings of this report are available from the corresponding author upon reasonable request.

\section{Consent}

The manuscript has been sufficiently anonymized, and no identifiable information has been included in the manuscript. Moreover, informed consent was obtained from the patient and her legal guardians.

\section{Disclosure}

Preliminary data of this case have been previously presented in the 2020 Lymphoma, Leukemia, and Myeloma Congress, October 21-24, 2020, in the abstract form.

\section{Conflicts of Interest}

The authors declare no conflicts of interest.

\section{Authors' Contributions}

EAP was responsible for clinical management of the patient and drafting the paper. RMN who is currently working as trainee in hematology and medical oncology has participated in the patient's management, performing literature research, and drafting the first manuscript. VY has kindly assisted RMN and provided significant input in the creation of the first draft of the manuscript. BS, DS, and CS have supervised the work, edited the content, and provided their expertise. All authors approved the final version of the manuscript submitted for publication.

\section{Acknowledgments}

The authors would like to thank all the Dr. Kariadi Transplant Unit personnel who were involved in patient care and Stem Cell Laboratory staff. Special acknowledgements belong to the patient and her family for accepting and consenting to the publication of this manuscript. Publication fee was granted by the Indonesian Society of Hematology and Medical Oncology of Semarang Chapter.

\section{References}

[1] M. Caballero, V. Rubio, J. Rifon et al., "BEAM chemotherapy followed by autologous stem cell support in lymphoma patients: analysis of efficacy, toxicity and prognostic factors," Bone Marrow Transplantation, vol. 20, no. 6, pp. 451-458, 1997.

[2] J. W. Sweetenham, "High dose chemotherapy and stem cell transplantation in Hodgkin's disease," in High Dose Chemotherapy: Principles and Practice, P. Lorigan and E. Vanderberghe, Eds., Martin Dunitz Ltd., London, UK, 2002.

[3] M. L. Gatesman and T. J. Smith, "The shortage of essential chemotherapy drugs in the United States," New England Journal of Medicine, vol. 365, no. 18, pp. 1653-1655, 2011.

[4] H. Hariman, "Thehematopoieticstemcelltransplantation inIndonesia: an unsolved dilemma," Bone Marrow Transplantation, vol. 42, no. Suppl. 1, pp. S85-S88, 2008.

[5] D. Santosa, E. A. Pangarsa, B. Setiawan et al., "Establishing the hematopoietic stem cell transplantation(HSCT)inadevelopingcountry;thejourneyofHSCT in Semarang, Indonesia," Bone Marrow Transplantation, vol. 56, no. Suppl. 1, pp. 1-4, 2020.

[6] A. Sureda and C. Martines, "Classical Hodgkin's lymphoma," in The EBMT Handbook: Hematopoietic Stem Cell Transplantation and Cellular Therapies, E. Carrera, C. Dufour, M. Mohty, and N. Kroger, Eds., Springer, Gewerbestrasse, Switzerland, 2019.

[7] A. Sureda, M. Constans, A. Iriondo et al., "Prognostic factors affecting long-term outcome after stem-cell transplantation in Hodgkin's lymphoma autografted after a first relapse," Annals of Oncology, vol. 16, no. 4, pp. 625-633, 2005.

[8] H. B. Wu, S. A. Yeh, and H. Y. Chen, "Brentuximab vedotin treatment for primary refractory Hodgkin lymphoma," Case Reports in Hematology, vol. 2013, Article ID 351292, 2013.

[9] D. C. Linch, D. Winfield, A. H. Goldstone et al., "Dose intensification with autologous bone-marrow transplantation in relapsed and resistant Hodgkin's disease: results of a BNLI randomised trial," The Lancet, vol. 341, no. 8852, pp. 1051-1054, 1993.

[10] N. Schmitz, B. Pfistner, M. Sextro et al., "Aggressive conventional chemotherapy compared with high-dose chemotherapy with autologous haemopoietic stem-cell transplantation for relapsed chemosensitive Hodgkin's disease: a randomised trial," The Lancet, vol. 359, no. 9323, pp. 2065-2071, 2002.

[11] K. K. Fields, G. J. Elfenbein, H. M. Lazarus et al., "Maximumtolerated doses of ifosfamide, carboplatin, and etoposide given over 6 days followed by autologous stem-cell rescue: toxicity profile," Journal of Clinical Oncology, vol. 13, no. 2, pp. 323-332, 1995.

[12] P. J. Stiff, S. Dahlberg, S. J. Forman et al., "Autologous bone marrow transplantation for patients with relapsed or 
refractorydiffuse aggressive non-Hodgkin's lymphoma: value of augmented preparative regimens--a Southwest Oncology Group trial," Journal of Clinical Oncology, vol. 16, no. 1, pp. 48-55, 1998.

[13] A. Colita, A. Colita, H. Bumbea et al., "LEAM vs. BEAM vs. CLV conditioning regimen for autologous stem cell transplantation in malignant lymphomas. Retrospective comparison of toxicity and efficacy on 222 patients in the first 100 days after transplant, on behalf of the Romanian Society of Bone Marrow Transplantation," Frontiers in Oncology, vol. 9, no. 892, pp. 1-10, 2019.

[14] J. M. Vose, S. Carter, L. J. Burns et al., "Phase III randomized study of rituximab/carmustine, etoposide, cytarabine, and melphalan (BEAM) compared with iodine-131 tositumomab/ BEAM with autologous hematopoietic cell transplantation for relapsed diffuse large B-cell lymphoma: results from the BMT CTN 0401 trial," Journal of Clinical Oncology, vol. 31, no. 13, pp. 1662-1668, 2013.

[15] J.-C. Jo, B. W. Kang, G. Jang et al., "BEAC or BEAM high-dose chemotherapy followed by autologous stem cell transplantation in non-Hodgkin's lymphoma patients: comparative analysis of efficacy and toxicity," Annals of Hematology, vol. 87, no. 1, pp. 43-48, 2008.

[16] E. Jantunen, T. Kuittinen, and T. Nousiainen, "BEAC or BEAM for high-dose therapyin patients with non-Hodgkin's lymphoma? A single centre analysis on toxicity and efficacy," Leukemia and Lymphoma, vol. 44, no. 7, pp. 1151-1158, 2003.

[17] Y.-B. Chen, A. A. Lane, B. R. Logan et al., "Impact of conditioning regimen on outcomes for patients with lymphoma undergoing high-dose therapy with autologous hematopoietic cell transplantation," Biology of Blood and Marrow Transplantation, vol. 21, no. 6, pp. 1046-1053, 2015.

[18] E. H. Wang, Y. A. Chen, S. Corrigan et al., "High-dose CEB vs BEAM with autologous stem cell transplant in lymphoma," Bone Marrow Transplantation, vol. 34, no. 7, pp. 581-587, 2004.

[19] J. Loke, J. Ward, P. Mahendra, S. Chaganti, and R. Malladi, "Outcomes of EAM conditioned autologous haematopoietic SCT for lymphoma: a matched pair retrospective single-centre study analysis," Bone Marrow Transplantation, vol. 48, no. 11, pp. 1486-1487, 2013.

[20] M. A. Bekadja, S. Talhi, K. Amani et al., "Outcomes of modified-eam conditioned autologous non-cryopreserved hematopoietic hematopoietic sct for lymphoma. A retrospective single-centre study," Bone Marrow Transplantation, vol. 53, no. 12, pp. 1596-1598, 2018.

[21] G. C. Seval, D. G. Sahin, E. A. Soydan et al., "Comparing conditioning remigens for autologous stem cell transplantation in lymphoma patients," Clinical Lymphoma, Myeloma \& Leukemia, vol. 18, no. Suppl. 1, p. S306, 2018.

[22] F. Abdel-Rahman, A. Hussein, M. Aljamily, A. Al-Zaben, N. Hussein, and A. Addasi, "High-dose therapy and autologous hematopoietic progenitor cells transplantation for recurrent or refractory Hodgkin's lymphoma: analysis of King Hussein Cancer Center results and prognostic variables," ISRN Oncology, vol. 12, no. 3, Article ID 249124, 2012.

[23] A. Josting, U. Rueffer, J. Franklin, M. Sieber, V. Diehl, and A. Engert, "Prognostic factors and treatment outcome in primary progressive Hodgkin lymphoma: a report from the German Hodgkin Lymphoma Study Group," Blood, vol. 96, no. 4, pp. 1280-1286, 2000.

[24] E. Van Den Neste, O. Cassasnovas, M. Andre et al., "Classical Hodgkin's lymphoma: the Lymphoma Study Association (LYSA) guidelines for relapsed and refractory adult patients eligible for transplant," Haematologica, vol. 98, no. 8, pp. 1-12, 2013.

[25] C. H. Moskowitz, J. Walewski, A. Nademanee et al., "Five-year PFS from the AETHERA trial of brentuximab vedotin for Hodgkin lymphoma at high risk of progression or relapse," Blood, vol. 132, no. 25, pp. 2639-2642, 2018. 